\title{
The Electrodynamics of Organic Superconductors
}

\author{
M. DRESSEL ${ }^{1}$, L. DEGIORGI ${ }^{1,2}$, O. KLEIN ${ }^{1}$ and G. GRÜNER ${ }^{1}$ \\ ${ }^{1}$ Department of Physics and Solid State Science Center, \\ University of California, Los Angeles, California 90024-1547 \\ ${ }^{2}$ Laboratorium für Festkörperphysik, \\ Eidgenössische Technische Hochschule, CH-8093 Zürich, Switzerland
}

\begin{abstract}
The electrodynamics of two classes of organic superconductors, based on the molecule BEDT-TTF, and on $\mathrm{C}_{60}$, is discussed. For both groups of materials, in the normal state the frequency dependent conductivity is that of a weakly correlated metal (with an interband transition in the infrared region). We find that for representative examples of both groups of organic materials the electrodynamics of the superconducting state is in full agreement with s-wave pairing.
\end{abstract}

Keywonds: Organic superconductors, Fullerenes, BEDT-TTF salts, electrodynamics, normal state properties, superconducting properties, optical and microwave investigations.

\section{Introduction}

Two groups of organic solids, one based on the molecule BEDT-TTF and one on $\mathrm{C}_{60}$, have attracted ample interst recently, mainly because of their high superconducting transition temperatures. At least twenty superconductors of BEDT-TTF salts have now been synthesized with $\kappa$-(BEDT-TTF $)_{2}\left[\mathrm{Cu}(\mathrm{NCS})_{2}\right], \kappa-(\mathrm{BEDT}-\mathrm{TTF})_{2} \mathrm{Cu}\left[\mathrm{N}(\mathrm{CN})_{2}\right]$ $\mathrm{Br}$ and $\kappa$-(BEDT-TTF $)_{2} \mathrm{Cu}\left[\mathrm{N}(\mathrm{CN})_{2}\right] \mathrm{Cl}$ undergoing the superconducting transition above $10 \mathrm{~K}$. On the side of the Buckminster Fullerenes, the transition temperatures of the dozen or so compounds already synthesized span the range from 2 to $33 \mathrm{~K}$. In both cases, large molecules with significant internal degrees of freedom are coupled forming relatively narrow bands with low electron density. The low carrier density and high superconducting transition temperature places these materials into the often called 'novel' superconductors together with the oxides and Chevrel phases as prime examples.

We will summarize the salient features of the electrodynamics of the normal and of the superconducting state of these materials.

\section{The Buckminster Fullerenes: $\mathbf{K}_{3} \mathrm{C}_{60}$ and $\mathbf{R b}_{3} \mathrm{C}_{60}$}

The electrical resistivity of a pressed pellet of $\mathrm{K}_{3} \mathrm{C}_{60}$ is shown in Fig. 1, measured by a contactless method at microwave frequencies. $\mathrm{K}_{3} \mathrm{C}_{60}$ exhibits a high conductivity 


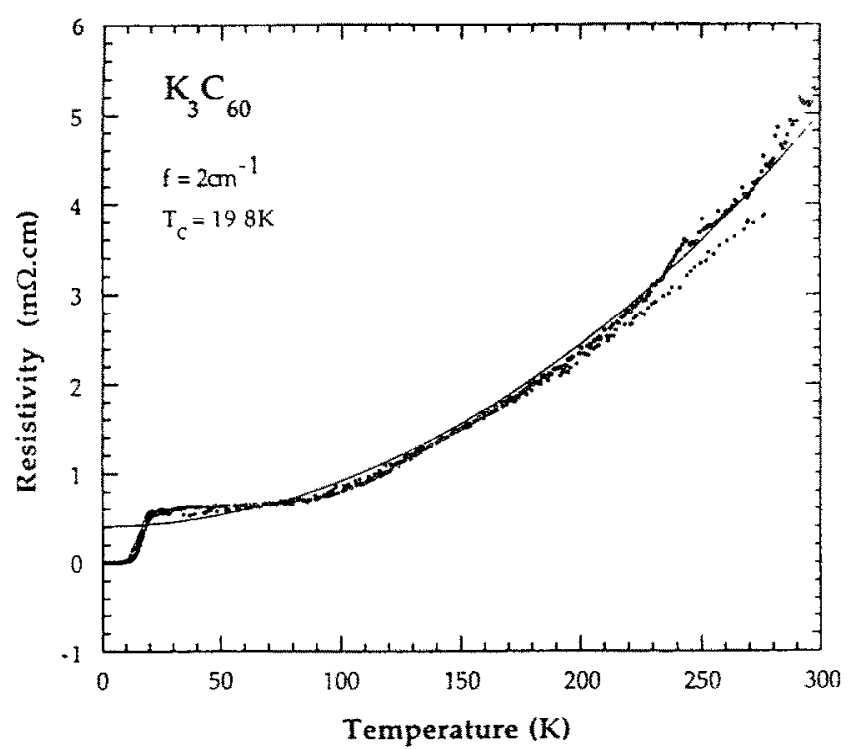

Figure 1: Temperature dependence of the intrinsic resistivity of $K_{3} C_{60}$. The solid line represents a fit of the data using the functional form $\rho(T)=0.41+5.1 \times 10^{-5} T^{2}$.

$\sigma(300 \mathrm{~K}) \approx 200(\Omega \mathrm{cm})^{-1}$ which increases approximately one order of magnitude right above $T_{c}=19.8 \mathrm{~K}$ (Klein et al, 1992); measurements on crystals and thin-films show a weaker temperature variation of about a factor 2 (Xiang et al. 1992, Palstra et al. 1992). $\mathrm{Rb}_{3} \mathrm{C}_{60}$ has similar properties with $\sigma(300 \mathrm{~K}) \approx 100(\Omega \mathrm{cm})^{-1}$ and $T_{c}=29 \mathrm{~K}$ (Haddon et al. 1991).

Reflection measurements on pressed $\mathrm{K}_{3} \mathrm{C}_{60}$ and $\mathrm{Rb}_{3} \mathrm{C}_{60}$ samples were performed between 14 and $50000 \mathrm{~cm}^{-1}$ using three different spectrometers. In the far infrared spectral range we measured $R(\omega)$ also as a function of temperature. After all measurements were performed, we covered our pellets with a $1000-\AA$ layer of gold in order to take into account the surface roughness (DeGiorgi et al. 1992).

\section{$2.1 \quad$ Normal State}

Figure 2 displays the optical conductivity $\sigma_{1}(\omega)$ at $300 \mathrm{~K}$ obtained from the KramersKronig (KK) analysis of our reflection data of $\mathrm{Rb}_{3} \mathrm{C}_{60}$ (DeGiorgi et al. 1992). We first note, that $\sigma_{1}(\omega)$ is remarkably different from that of a simple metal where the Drude model is appropriate and for which:

$$
\int_{0}^{\infty} \sigma_{1}(\omega) d \omega=\frac{\pi n e^{2}}{2 m_{b}}=\frac{\omega_{p}^{2}}{8}
$$

with $n$ the number of carriers, $m_{b}$ the band mass and $\omega_{p}$ the plasma frequency. Instead, we observe a Drude-like response - which, however, does not lead to the oscillator strength as given by Eq. 1 - together with a mid-infrared absorption, and several absorptions at higher frequencies due most probably to interband transitions. The overall behavior of $\sigma_{1}(\omega)$ is similar to that observed in BEDT-TTF salts (see below) and 


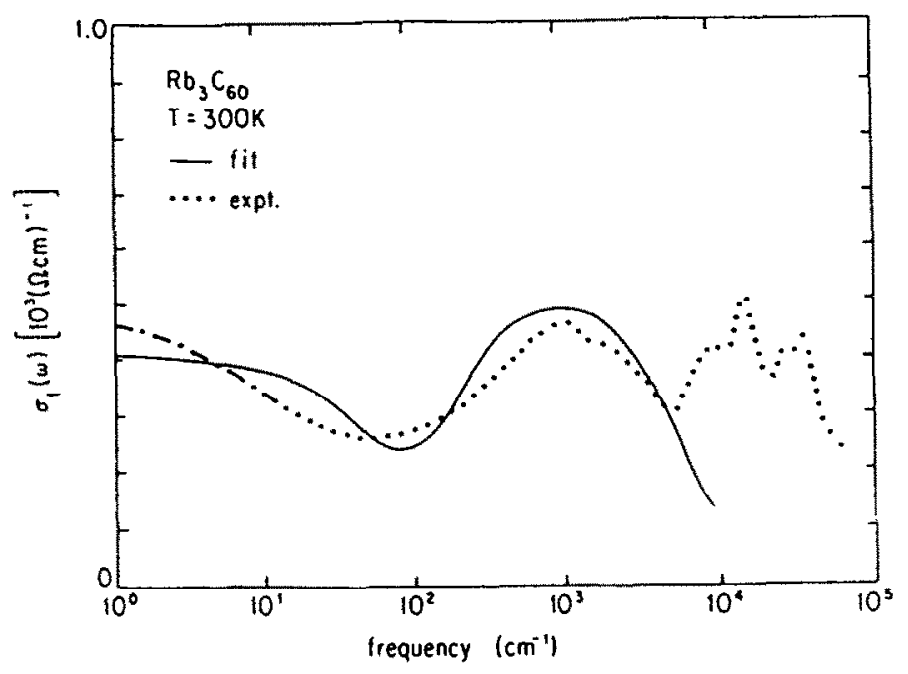

Figure 2: Optical conductivity of $R b_{3} C_{60}$ at $300 \mathrm{~K}$ as evaluated from $K K$ analysis. The solid line represents a phenomenological fit based on Eq. 2. The dot-dashed line shows the $K K$ result in the range of the Hagen-Rubens extrapolation.

various correlated materials with low electron density, notably the high- $T_{c}$ oxides and $\mathrm{BaBi}_{x} \mathrm{~Pb}_{1-x} \mathrm{O}_{3}$ (Tajima et al. 1985).

In the high frequency range from the visible to the ultra violet, our excitation spectrum is characterized by broad transition peaked at $18000 \mathrm{~cm}^{-1}$ and at $35000 \mathrm{~cm}^{-1}$. We ascribe these excitations to the interband transitions involving $s$ and $p$ carbon orbitals and $s, p$, and $d$ alkali orbitals. We remark that our preliminary measurements on the undoped $\mathrm{C}_{60}$ are also suggestive of interband transitions with different energy positions and oscillator strengths. Our measured conductivity is in good agreement with the theoretical predictions extracted from a band-structure calculation ( $\mathrm{Xu}$ et al. 1991), which lead to broad peaks centered at $1,2.5$, and $4 \mathrm{eV}$, and electron energy-loss studies (Sohmen and Fink 1993). This calculation also suggests that the electronic structure of the ternary alkali-metal-doped $\mathrm{C}_{60}$ compounds cannot be simply interpreted as the filling of the extra electrons in the lowest unoccupied conduction bands in the context of a rigid-band model. This has important consequences for the states very close to the Fermi energy $\epsilon_{F}$, as we will discuss below in detail. The theory also predicts weak optical electronic transitions [seen by energy-loss spectroscopy (Sohmen et al. 1992, Sohmen and Fink 1993)] above $4.5 \mathrm{eV}\left(\approx 40000 \mathrm{~cm}^{-1}\right)$, which, however, we do not observe, presumably due to the very low reflectivity in this frequency range.

The low-frequency part of $\sigma_{1}(\omega)$ [i.e., from the far infrared (FIR) up to the mid infrared (MIR)] is characterized by two main features: the broad band centered at approximately $1000 \mathrm{~cm}^{-1}$ and the Drude-like contribution to $\sigma_{1}(\omega)$ at FIR frequencies. In view of these features we model the dielectric function with the following expression:

$$
\epsilon(\nu)=\epsilon_{\infty}+\nu_{P}^{2}\left[\frac{f_{G}}{\nu_{G}^{2}-\nu^{2}-i \nu \gamma_{G}}-\frac{1-f_{G}}{\nu^{2}+i \nu \gamma_{D}}\right],
$$

where $\epsilon_{\infty}$ describes the high-frequency contribution to the dielectric function, while the second and third therm in the parentheses describe the MIR broad excitation (i.e., at 
$\approx 1000 \mathrm{~cm}^{-1}$ ) and the Drude-like response at low frequencies, repectively. The best fit as shown in Fig. 2 is obtained with the following parameters: $\epsilon_{\infty}=7, \nu_{P}=13000 \mathrm{~cm}^{-1}$, $\nu_{G}=1000 \mathrm{~cm}^{-1}, \gamma_{G}=6000 \mathrm{~cm}^{-1}, \gamma_{D}=60 \mathrm{~cm}^{-1}$, and $f_{G}=0.992$.

Two different approaches can be taken to interpret these findings. In a one-component picture we assume that the low-frequency Drude-like behavior and the harmonic oscillator at $1000 \mathrm{~cm}^{-1}$ are due to the response of the conduction band, and, crudely speaking, the charge carriers behave as free carriers at low frequencies and as bound ones at high frequencies. This interpretation is similar to that often applied to the high- $T_{c}$ $\mathrm{Cu}-\mathrm{O}$ superconductors and some heavy Fermion materials, where a simple Drude model also cannot describe $\sigma_{1}(\omega)$ (Tanner and Timusk 1992). Frequency dependent mass renormalization due to electron-electron interactions, and alternatively midgap states (like polarons or excitons) or a broad phonon spectrum (i.e., Holstein process) were suggested as a source of the frequency-dependent scattering at FIR and MIR frequencies. The total spectal weight associated with the plasma frequency $\omega_{p} / 2 \pi c=\nu_{p}=13471 \mathrm{~cm}^{-1}$ would correspond to an effective band mass $m_{b}=2 m_{e}$, assuming a charge carrier density $n=4.1 \times 10^{21} \mathrm{~cm}^{-3}$ from simple counting arguments. This value is somewhat smaller than the value arrived at from spin susceptibility measurements, $m_{b}=6.5 m_{e}$ (Wong et al. 1992), and from electron energy-loss spectroscopy, $m_{b}=4 m_{e}$ (Sohmen and Fink 1993).

The second way to interpret Eq. 2 is to consider the two contributions independently, using a two-component picture. Then, the Drude part is characterized by a plasma frequency $\nu_{p}=1205 \mathrm{~cm}^{-1}$ [i.e., $\left(1-f_{G}\right)^{1 / 2} \nu_{p}$ ], and the harmonic oscillator is viewed as a separate MIR excitation, in a fashion similar to the normal-state description currently used for high- $T_{c}$ superconductors (Tanner and Timusk 1992). First, we note that the scattering rate $\left(\gamma_{D}\right)$ of the Drude component is comparable to the superconducting gap $2 \Delta=60 \mathrm{~cm}^{-1}$ (see below). This suggests that the $\mathrm{Rb}_{3} \mathrm{C}_{60}$ compound is close to the clean limit. We suggest that the broad excitation at approximately $1000 \mathrm{~cm}^{-1}$ can be assigned to an electronic interband transition. This interpretation is particularly compelling in view of a recent result obtained with photoemission (XPS) and bremsstrahlung isochromat (inverse photoemission) (BIS) spectroscopy (Takahashi et al. 1992). These experiments indicate that alkali-metal-doping causes a transfer of electronic states from the lowest unoccupied molecular orbital $\left(t_{1 u}\right)$ band to an additional band produced in the band gap (of pure $\mathrm{C}_{60}$ ). A comparison of the XPS and BIS spectra indicate that a pseudogap of about $0.5 \mathrm{eV}\left(\approx 4000 \mathrm{~cm}^{-1}\right)$ opens at the Fermi level. Thus, our broad excitation peak around $0.1 \mathrm{eV}$ may be considered as the optical evidence of such a pseudogap, and this interpretation would also agree with our observation of a small oscillator strength in the Drude term $\left(\nu_{P}=1205 \mathrm{~cm}^{-1}\right)$. Recent calculations (Huang et al. 1992) of the $\mathrm{Rb}_{3} \mathrm{C}_{60}$-band structure indicate that the density of states of the metallic band peaks $0.13 \mathrm{eV}$ above the Fermi level; therefore intraband transitions between these states are likely. Nevertheless, there are some severe problems also with this two-component picture. In fact, the small plasma frequency associated with the Drude response of the free charge carriers would imply a large effective band mass or a smaller effective number of carriers $\left(n_{e f f}<n=4.1 \times 10^{21} \mathrm{~cm}^{-3}\right)$, and moreover a very large penetration depth compared to the experimental one (Wong et al. 1992). 


\subsection{Superconducting State}

Next we turn to the superconducting state of the Buckminster Fullerenes. The muon spin relaxation experiments of the Columbia group, made on a $\mathrm{K}_{3} \mathrm{C}_{60}$ samples prepared at UCLA give the temperature dependent penetration depth as displayed in Fig. 3. Both, these results (Uemura et al. 1991) and our earlier experiments (Holczer et al. 1991, Sparn et al. 1992) on $H_{c 1}$ and $H_{c 2}$ strongly suggest $s$-wave pairing.

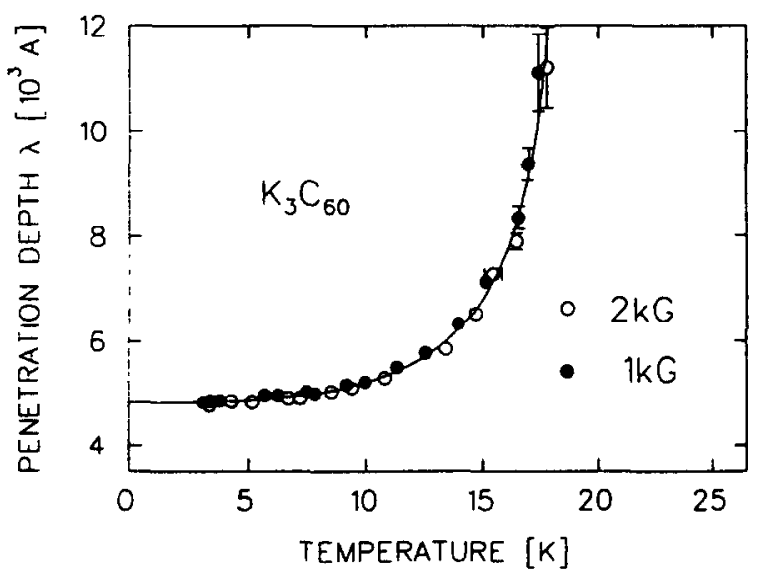

Figure 3: The magnetic field penetration depth $\lambda(T)$ in $K_{3} C_{60}$ derived from $\mu S R$ measurements.

In Fig. 4 we display the optical reflectivity $R(\omega)$ of both compounds $\mathrm{K}_{3} \mathrm{C}_{60}$ and $\mathrm{Rb}_{3} \mathrm{C}_{60}$ measured a different temperatures above and well below the superconducting transition $T_{c}$ in the spectral range which covers the superconducting gap. As a result of the KK analysis, $\sigma_{1}(\omega)$ is shown in Figure 5. The low-frequency extrapolation of $\sigma_{1}(\omega)$ (dashed-double dotted line) is that of the Hagen-Rubens form in the normal state, and this leads to a conductivity $\sigma(\omega \rightarrow 0)=n e^{2} \tau / m$ of $1.2 \times 10^{3}(\Omega \mathrm{cm})^{-1}$ and $1.3 \times 10^{3}(\Omega \mathrm{cm})^{-1}$ for $\mathrm{Rb}_{3} \mathrm{C}_{60}$ and $\mathrm{K}_{3} \mathrm{C}_{60}$, respectively, in full agreement with the dc (Palstra et al. 1991, Xiang et al. 1992), or our microwave (Fig. 1), resistivity
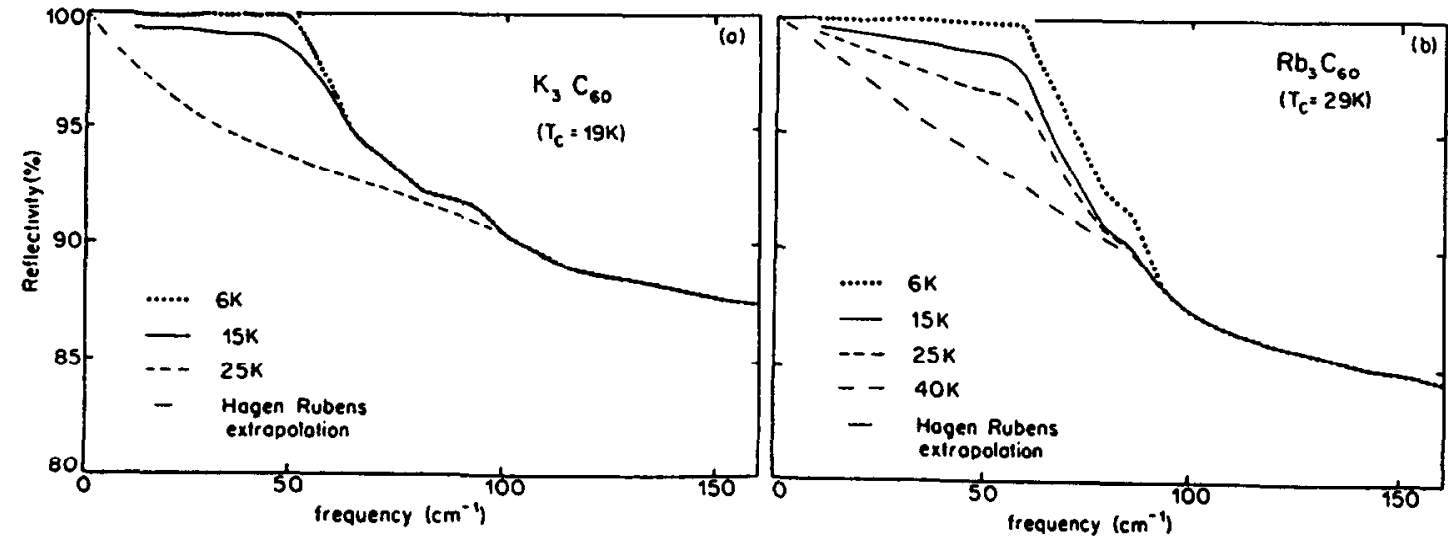

Figure 4: The optical reflectivity above and below $T_{c}$ for (a) $K_{3} C_{60}$ and (b) $R b_{3} C_{60}$. 


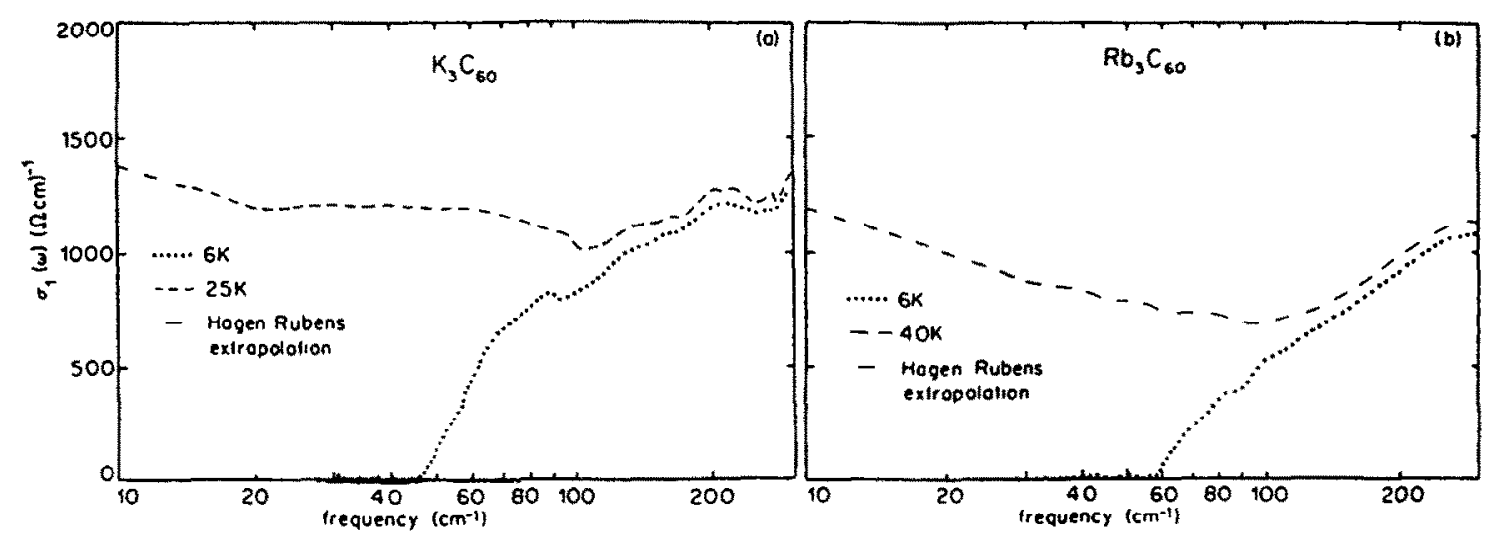

Figure 5: The optical conductivity above and below $T_{c}$ for (a) $K_{3} C_{60}$ and (b) $R b_{3} C_{60}$ as evaluated from $K K$ analysis of the reflectivity data.

values. In the superconducting state at $T=6 \mathrm{~K}$, our reflectivity data are, up to a well-defined threshold frequency of $48 \mathrm{~cm}^{-1}$ for $\mathrm{K}_{3} \mathrm{C}_{60}$ and $60 \mathrm{~cm}^{-1}$ for $\mathrm{Rb}_{3} \mathrm{C}_{60}$, within the experimental error of $0.5 \%$ equal to 1 , and we have used this value to evaluate the optical conductivity. This procedure gives, for both compounds, zero conductivity up to a threshold frequency $\nu_{\text {gap }}$ which we identify as the superconducting gap, and we obtain $\Delta=24 \mathrm{~cm}^{-1}$ for the $\mathrm{K}$ and $30 \mathrm{~cm}^{-1}$ for the $\mathrm{Rb}$ compound. Together with the superconducting transition temperature $T_{c}=19$ and $29 \mathrm{~K}$ this leads to the ratio $2 \Delta / k_{B} T_{c}=3.6$ and 2.98 for $\mathrm{K}_{3} \mathrm{C}_{60}$ and $\mathrm{Rb}_{3} \mathrm{C}_{60}$, with both values in good agreement with the weak-coupling BCS results of $2 \Delta / k_{B} T_{c}=3.52$. Due to the complicated frequency dependence of $\sigma_{1}$ in the region of the gaps, the conventional arguments, which relate the mean free path $l$ and coherence length $\xi$ to the penetration depth $\lambda$ (the usual Pippard correction Eq. 13), do not apply. However, our conductivity results, evaluated as discussed above can be compared with the measured penetration depth values (Uemura et al. 1991, Uemura et al 1992) using the missing spectral weight argument (Tinkham 1975). The penetration depth $\lambda$ is given by

$$
\lambda=c\left[8 \int_{0}^{\infty}\left[\sigma_{1, n}(\omega)-\sigma_{1, s}(\omega)\right] d w\right]^{-1 / 2},
$$

where $\sigma_{1, n}$ and $\sigma_{1, s}$ are the optical conductivity in the normal and superconducting state, respectively. We performed the integral up to the frequency $\nu=350 \mathrm{~cm}^{-1}$ where $\sigma_{1, n}$ and $\sigma_{1, s}$ are no longer different within the experimental error of our measurement and we obtain $\lambda=8000 \pm 500 \AA$ for the two compounds, in satisfatory agreement with the penetration depth of $6000 \AA$ (Tycko et al. 1992) or $4800 \AA$ (Uemura et al. 1991) on $\mathrm{K}_{3} \mathrm{C}_{60}$ and $4600 \AA$ (Tycko et al. 1992) or $4200 \AA$ (Uemura et al. 1992) for $\mathrm{Rb}_{3} \mathrm{C}_{60}$.

The functional form of $\sigma_{1}(\omega)$ has been evaluated first by Mattis and Bardeen (1958), and this theory leads to

$$
\begin{aligned}
\frac{\sigma_{1, s}(\omega, T)}{\sigma_{n}}= & \frac{2}{\hbar \omega} \int_{\Delta}^{\infty}[f(E)-f(E+\hbar \omega)] g(E) d E \\
& +\frac{1}{\hbar \omega} \int_{\Delta-\hbar \omega}^{-\Delta}[1-2 f(E+\hbar \omega)] g(E) d E
\end{aligned}
$$



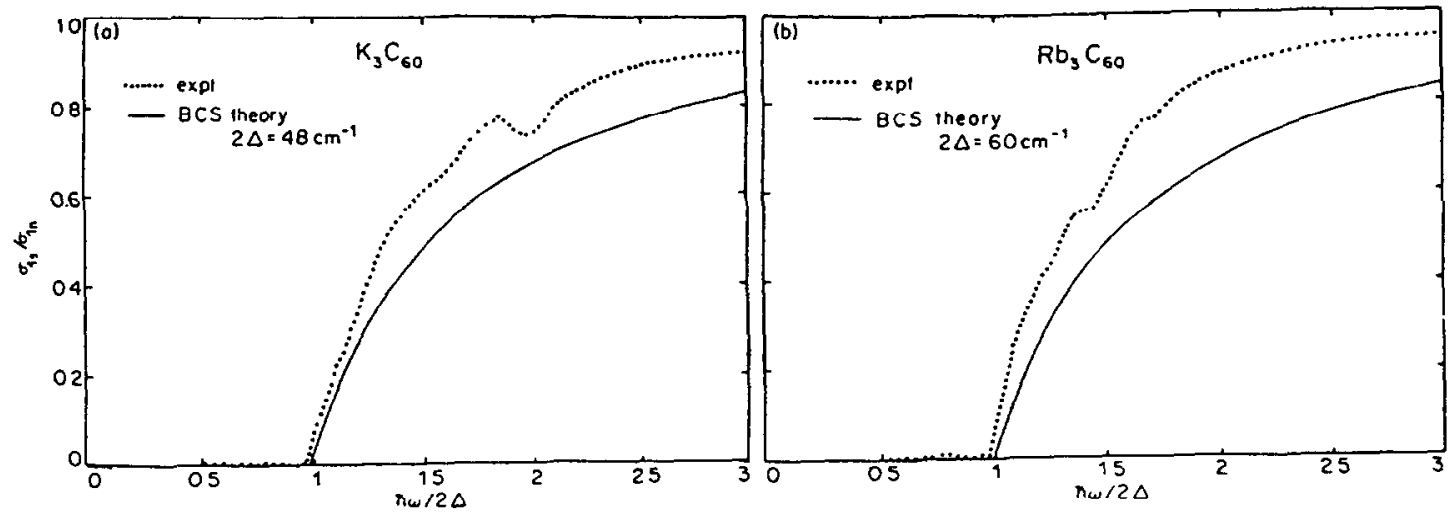

Figure 6: The measured optical conductivity $\sigma_{1, s}(\omega) / \sigma_{1, n}$ for (a) $K_{3} C_{60}$ and (b) $R b_{3} C_{60}$ together with the BCS behavior calculated using the Mattis-Bardeen formula.

$$
\frac{\sigma_{2, s}(\omega, T)}{\sigma_{n}}=\frac{1}{\hbar \omega} \int_{\Delta-\hbar \omega,-\Delta}^{\Delta} \frac{[1-2 f(E+\hbar \omega)]\left(E^{2}+\Delta^{2}+\hbar \omega E\right)}{\left(\Delta^{2}-E^{2}\right)^{1 / 2}\left[(E+\hbar \omega)^{2}-\Delta^{2}\right]^{1 / 2}} d E
$$

where

$$
\begin{aligned}
& f(E)=\frac{1}{e^{E / k_{B} T}+1} \\
& g(E)=\frac{\left(E^{2}+\Delta^{2}+\hbar \omega E\right)}{\left(E^{2}-\Delta^{2}\right)^{1 / 2}\left[(E+\hbar \omega)^{2}-\Delta^{2}\right]^{1 / 2}}
\end{aligned}
$$

and the limits of integration are those specified in the original paper. The only input parameters in these calculations are the values of the energy gap $\Delta$, the temperature $T$, and the frequency $\omega$. Equation 4 in the $T \rightarrow 0$ limit, together with the measured optical conductivity, is displayed for both compounds in Fig. 6. Given the fact that the calculation neglects the role played by mean free path effects, the agreement between our results and the theory based on a BCS ground state is excellent. We also note that the functional form also reflects the so-called case II coherence factors (Tinkham 1975) which depend sensitively on the symmetry of the superconducting wave function, and therefore the agreement between theory and experiment gives clear evidence for a singlet ground state.

The observation of a well-defined superconducting gap would suggest that, strictly, speaking these materials are not in the so-called clean limit, where the mean free path $l$ exceeds the coherence length $\xi$. This issue has been adressed before (Palstra et al. 1991, Xiang et al. 1992, Sparn et al. 1992, Holczer et al. 1991) and is the subject of acute controversy. From measurements of the lower and upper critical field $H_{c, 1}(T)$ and $H_{c, 2}(T)$ the zero-temperature coherence length $\xi_{0}$ can be evaluated to $26 \AA$ and $20 \AA$ for $\mathrm{K}_{3} \mathrm{C}_{60}$ and $\mathrm{Rb}_{3} \mathrm{C}_{60}$, respectively (Holczer et al. 1991, Sparn et al. 1992), while we estimated $l=280 \AA$ for the mean free path at room temperature. The question of whether the clean or the dirty limit applies is based on the assumption that a simple Drude response accounts for the conductivity in the metallic state. We have shown above, and it is also evident from Fig. 6 , that $\sigma_{1}(\omega)$ cannot be described by the Drude model alone, but can be accounted for by a low frequency Drude response and a MIR 
absorption (Eq. 2). Both features contribute, within the one component picture of the normal state, to the total spectral weight

$$
\int_{0}^{\omega_{c}} \sigma_{1}(\omega) d \omega=\frac{\pi n e^{2}}{2 m_{b}}
$$

with $\omega_{c}$ a cutoff frequency of the order of $10^{4} \mathrm{~cm}^{-1}$, chosen so as to include contributions from the band which intersects the Fermi level, but excluding the high-lying bands. Therefore, in order to evaluate the various parameters of the superconducting state as the London penetration depth $\lambda_{L}$, or the coherence length $\xi$ in terms of the normal state properties (given in the Drude model by the single parameter $l$, or equivalently $\tau$ ), a more elaborate analysis is necessary.

\section{The Layered Organic Superconductors: (BEDT-TTF) ${ }_{2}-$ $\left[\mathrm{Cu}(\mathrm{NCS})_{2}\right]$}

The Buckminster Fullerenes crystalize in a isotropic three-dimensional structure with the hollow carbon-net structures of $\mathrm{C}_{60}$ forming a face centered cubic lattice and the alkali-ions occupying the octahedral and tetrahedral vacancies. BEDT-TTF salts contain the almost plane molecules $\mathrm{S}_{8} \mathrm{C}_{10} \mathrm{H}_{4}$ which in the $\kappa$-phase are arranged in dimer pairs with alternating orientations. The BEDT-TTF molecules form conducting sheets in the (bc)-plane separated by polymeric anions $\mathrm{Cu}(\mathrm{NCS})_{2}^{-}$(Williams et al. 1992). These layered compounds show structural similarities to the high- $T_{c}$ oxide superconductors. The room temperature conductivity is about $20(\Omega \mathrm{cm})^{-1}$ with an in-plane anisotropy $\sigma_{c} / \sigma_{b}$ of approximately a factor 2 and an out-of-plane anisotropy $\sigma_{c} / \sigma_{a}$ larger than 5000 . In Fig. 7 we display the resistivity evaluated from microwave measurements at $35 \mathrm{GHz}$ together with $\rho_{d c}$; the data are normalized and $\sigma_{d c}(T=12 \mathrm{~K})=3.8 \times 10^{3}(\Omega \mathrm{cm})^{-1}$.

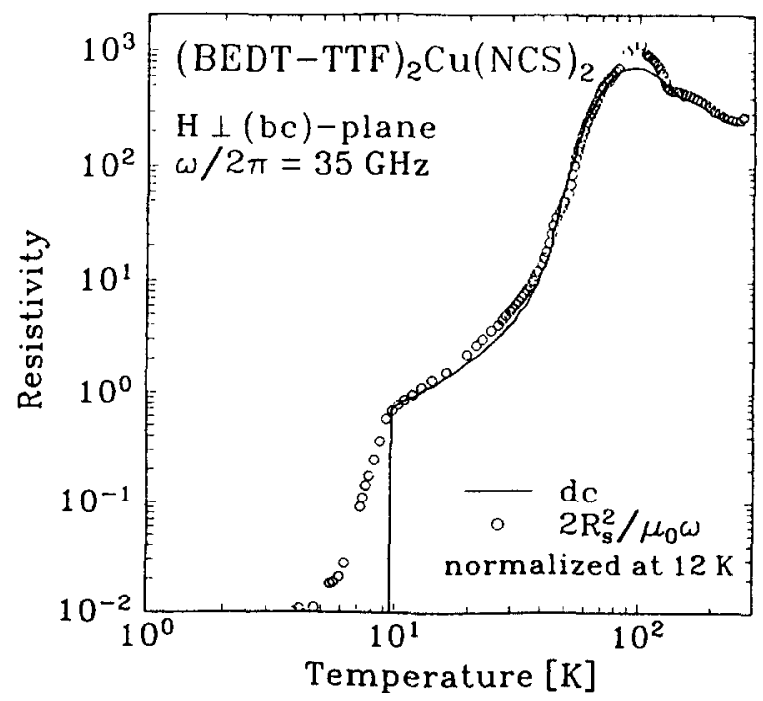

Figure 7: The temperature dependence of the $d c$ resistivity and $2 R_{S}^{2} / \mu \omega$ for $\kappa-(B E D T$ $\mathrm{TTF})_{2}\left[\mathrm{Cu}(\mathrm{NCS})_{2}\right]$. All quantities are normalized to the their $\mathrm{T}=12 \mathrm{~K}$ value. 


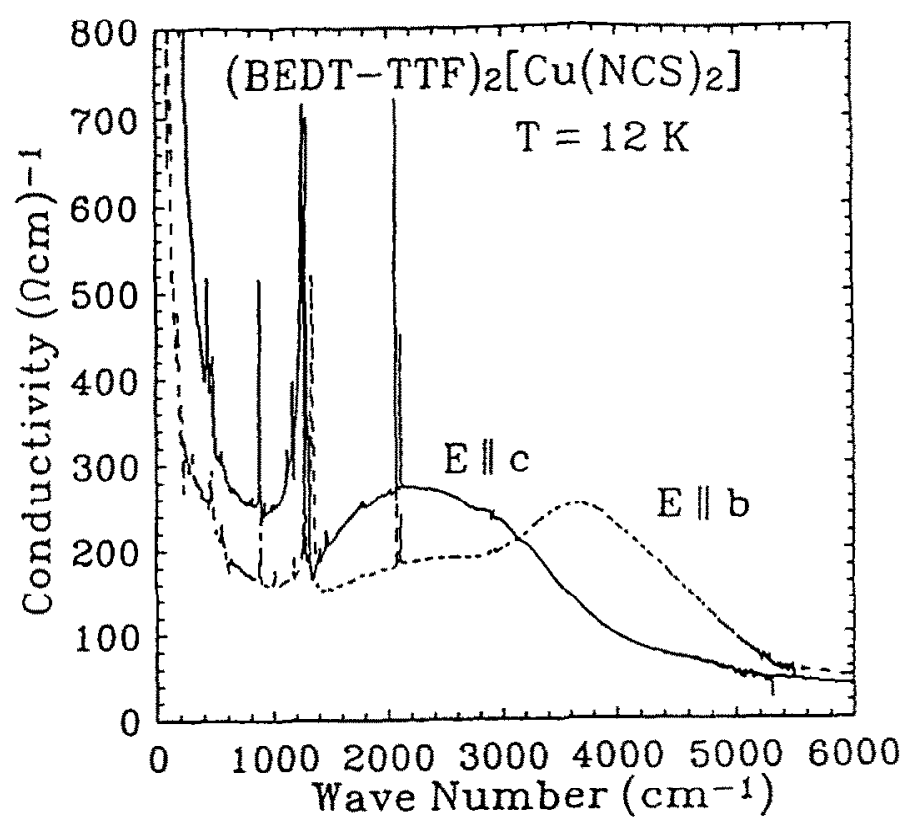

Figure 8: The optical conductivity $\sigma(\omega)$ of (BEDT-TTF $)_{2}\left[C u(N C S)_{2}\right]$ for $\vec{E} \| b$ and $\vec{E} \| c$ as evaluated from reflectivity measurements at $T=12 \mathrm{~K}$ by $\mathrm{KK}$ analysis. (After Kornelsen et al. 1989)

The superconducting transition is as high as $10.4 \mathrm{~K}$ for the resistivity midpoint and $T_{c}$ $\approx 8.6 \mathrm{~K}$ from ac-susceptibility measurements with a broad transition of more than $1 \mathrm{~K}$.

\subsection{Normal State}

Extensive investigations of the optical properties of $\kappa$-(BEDT-TTF $)_{2}\left[\mathrm{Cu}(\mathrm{NCS})_{2}\right]$ single crystals have been conducted by John Eldridge's group (Kornelsen et al. 1989, Kornelsen et al. 1991). The reflectivity in both directions of the conducting plane was measured at various temperatures in the frequency range from $200 \mathrm{~cm}^{-1}$ to $8000 \mathrm{~cm}^{-1}$. As a result of the KK analysis the optical conductivity $\sigma(\omega)$ in the normal state is displayed in Fig. 8.

Neither the high nor the low-temperature results follow a simple Drude behavior. However, the MIR reflectivities of the organic conductor have a basic shape somewhat similar to that derived from a simple Drude response, and $\epsilon(\nu)=\epsilon_{\infty}-\nu_{p}^{2} /\left(\nu^{2}+i \nu \gamma\right)$ can be applied to obtain estimates for $\epsilon_{\infty}, \nu_{p}$ and $\gamma$. The fit describes the $T=12 \mathrm{~K}$ reflectivity data sufficiently only at frequencies above $3500 \mathrm{~cm}^{-1}$. It leads to $\nu_{p}=8730 \mathrm{~cm}^{-1}, \gamma=$ $1260 \mathrm{~cm}^{-1}$ and $\epsilon_{\infty}=3.37$ in the $b$-direction, and to $\nu_{p}=8520 \mathrm{~cm}^{-1}, \gamma=1195 \mathrm{~cm}^{-1}$ and $\epsilon_{\infty}=4.13$ for $\vec{E} \| c$. Assuming a carrier density $n=1.23 \times 10^{21} \mathrm{~cm}^{-3}$ resulting from one charge per two BEDT-TTF molecules, the effective mass can be calculated to $m_{b}=1.5 m_{e}$. Looking at the zero-crossing of the dielectric constant $\epsilon^{\prime}, \nu_{p}=3840 \mathrm{~cm}^{-1}$ and $7490 \mathrm{~cm}^{-1}$ for $\vec{E} \| b$ and $\vec{E} \| c$, respectively, can be estimated, assuming the $\epsilon_{\infty}$ values obtained above. These values of the plasma frequency are similar to results obtained from the peak of the loss function $-1 / \operatorname{Im}(\epsilon)$ and from sum-rule arguments (Eq. 1) (Kornelsen et al. 1989). 
The overall conductivity spectrum can be interpreted as a combination of a FIR contribution from intraband transitions and a MIR interband contribution. The MIR band is at a higher frequency for $\vec{E} \| b$ than for $\vec{E} \| c$ and, due to thermal contraction of the lattice upon cooling, moves even higher for $\vec{E} \| b$ while becoming weaker for $\vec{E} \| c$. It might be looked at in two different ways: it can be seen as due to a charge-transfer between the dimers, or with the help of the band structure as calculated by Jung et al. (1989) and Saito (1990). Interband transitions will take place vertically from the filled bands below the Fermi level to the empty ones above. Broad peaks in the conductivity spectra are expected where the density of states is high. An intersection with the Fermi level is found at $2250 \mathrm{~cm}^{-1}$ in the $c$-direction and at approximately $3350 \mathrm{~cm}^{-1}$ for $\vec{E} \| b$. This is in good agreement with the observed peaks in the optical conductivity $\left(2200 \mathrm{~cm}^{-1}\right.$ for $\vec{E} \| c$ and $3500 \mathrm{~cm}^{-1}$ for $\vec{E} \| b$ ) as displayed in Fig. 8 (Kornelsen et al. 1991).

In order to fit the obtained optical conductivity in Fig. 8 we generalize the DrudeLorentz model of Equation 2:

$$
\epsilon(\nu)=\epsilon_{\infty}+\sum_{i} \frac{\nu_{p i}^{2}}{\nu_{0 i}^{2}-\nu^{2}-i \nu \gamma_{i}},
$$

and take more terms into account $\left(\nu_{0 i}=0\right.$ describes a Drude term and $\nu_{0 i} \neq 0$ a Lorentz oscillator). A good fit requires two strongly damped Lorentz terms in the MIR, a broad Drude term giving the shoulder at $400 \mathrm{~cm}^{-1}$ and the low frequency strength up to $1200 \mathrm{~cm}^{-1}$, and, most important, a second Drude term giving the narrow $\mathrm{dc}$ conductivity peak. One finds that at low frequencies a renormalized Drude response occurs, with an effective mass $m_{b} / m_{e}=8$ for $\vec{E} \| b$ and 5 in the $c$-direction; and $\gamma$ $=40 \mathrm{~cm}^{-1}$ and $80 \mathrm{~cm}^{-1}$, respectively. Comparing with the values obtained from the reflectivity fit $\left(\gamma \approx 1200 \mathrm{~cm}^{-1}\right)$ a frequency dependent scattering seems to be likely. This behavior is confirmed by our investigations of the surface impedance in the millimeter wave range (see below), where $1 / \tau=15 \mathrm{~cm}^{-1}$ was obtained at $f=60 \mathrm{GHz}=2 \mathrm{~cm}^{-1}$. From Shubnikov-de-Haas measurements $1 / \tau=2 \mathrm{~cm}^{-1}$ is evaluated (Toyota et al. 1988).

\subsection{Superconducting State}

Far-infrared absorption measurements down to $\nu=10 \mathrm{~cm}^{-1}$ (Kornelsen et al. 1990) on $\kappa$-(BEDT-TTF $)_{2}\left[\mathrm{Cu}(\mathrm{NCS})_{2}\right]$ in the superconducting state were not able to identify the superconducting gap, which is expected at about $\nu_{\text {gap }}=2 \Delta(T=0)=3.5 k_{B} T_{c}=$ $25 \mathrm{~cm}^{-1}$. Measurements in the millimeter wave spectral range up to $f=102 \mathrm{GHz} \approx$ $3 \mathrm{~cm}^{-1}$ (Holzcer et al. 1990), however, clearly show a change in the surface resistance at $T_{c}$. This may indicate that the material is in the clean limit, where the optical gap is hard to detect. The scattering rate of $\gamma \approx 1000 \mathrm{~cm}^{-1}$ as obtained from the MIR fit (Kornelsen et al. 1989) of the reflectivity does not describe the FIR properties where the superconducting gap is located, and values for the scattering rate $\gamma$ of less than $20 \mathrm{~cm}^{-1}$ as given by Shubnikov-de-Haas experiments or microwave measurements are more suitable.

Various experiments in the superconducting state of BEDT-TTF materials indicate deviations from what is expected for conventional singlet pairing. The temperature dependence of the critical field, NMR relaxation time, and the anomalous behavior of the 
specific heat have been interpreted in terms of triplet pairing, spin density wave transition below $T_{c}$ and vortex glass transition as expected for a ground state with higher momentum pairing (Oshima et al. 1988, Katsumoto et al. 1988, Takahashi et al. 1988). While both NMR and specific heat measurements are sensitive also to excitations other than those of the superconducting state, the parameters which characterize the electrodynamics, the penetration depth $\lambda$ and surface resistance $R_{S}$ are free from such complications. Consequently, the magnitude and temperature dependence of these parameters may in principle distinguish between the various symmetries of superconducting states. Recent experiments employing muon spin rotation are controversial, one (Harshman et al. 1990) suggests $s$-wave pairing, the other (Le et al. 1992) shows important deviations from the BCS behavior. Low frequency ac magnetization measurements (Kanoda, et al. 1990) with $H_{a c}$ parallel to the layers led to an unusually large penetration depth along the $(b c)$-plane, and a temperature dependence suggesting higher momentum pairing.

We have conducted experiments on the low frequency $(\hbar \omega<\Delta)$ electrodynamical response in the normal and superconducting state of (BEDT-TTF $)_{2}\left[\mathrm{Cu}(\mathrm{NCS})_{2}\right]$, by measuring the the surface impedance $Z_{S}$ at millimeter wave frequencies (i.e., 35 up to $100 \mathrm{GHz}$ ) down to $T=0.8 \mathrm{~K}$. The surface impedance is defined at

$$
Z_{S}=\frac{E_{0}}{\int_{0}^{\infty} j d x}
$$

where $E_{0}$ is the electric field at the surface, $j$ is the ac current in the sample and the $x$ direction is normal to the surface. $Z_{S}$ is, in terms of the complex conductivity $\sigma=\sigma_{1}+i \sigma_{2}$, given by

$$
Z_{S}=R_{S}+i X_{S}=\left(\frac{i \mu_{0} \omega}{\sigma_{1}-i \sigma_{2}}\right)^{1 / 2}
$$

where $R_{S}$ and $X_{S}$ are the surface resistance and surface reactance, respectively. In the normal state, at frequencies $\omega \tau<1, \sigma_{1} \ll \sigma_{2}$ and consequently

$$
R_{S}=X_{S}=\left(\frac{\mu_{0} \omega}{2 \sigma_{d c}}\right)^{1 / 2}
$$

i.e., the surface resistance is equal to the surface reactance in the so-called Hagen Rubens limit, and $\sigma_{1}(\omega \ll 2 \pi / \tau) \approx \sigma_{d c}$. Consequently, at low frequencies the resistivity is given by $\rho(T)=2 R_{S}^{2} / \mu_{0} \omega$.

In the superconducting state well below $T_{c}$ the surface reactance is given by

$$
X_{S}(T)=\mu_{0} \omega \lambda(T)
$$

where the surface reactance $X_{S}(T)$, which is proportional to the measured frequency shift, is proportional to the penetration depth $\lambda$ and therefore can be directly compared with various models of the superconducting state.

Two configurations have been employed. In one arrangement the ac magnetic field is directed perpendicular to the highly conducting (bc)-plane. Consequently in this configuration the penetration depth in the planes $\lambda_{\|}$is measured. In another configuration, the ac magnetic field is parallel to the direction, i.e., with the highly conducting (bc)-plane. In this configuration both the penetration depth along the layers $\lambda_{\|}$and perpendicular 


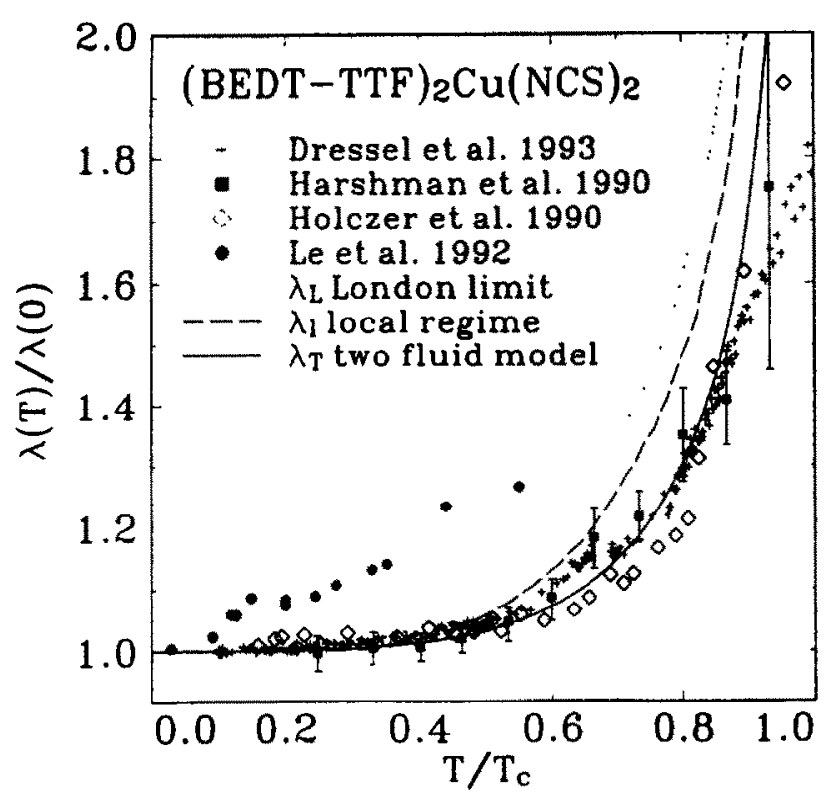

Figure 9: Temperature dependence of the penetration depth both parallel (Dressel et al. 1993) and perpendicular (Holczer et al. 1990) to the layers. $\lambda_{\|}=1 \mu \mathrm{m}$ and $\lambda_{\perp}=$ $40 \mu \mathrm{m}$ as $T \rightarrow 0$. The $\mu S R$ experiments of two different goups (Harshman et al. 1990, Le et al. 1992) are shown as well. The dotted line is the BCS weak coupling limit, the dashed line represents the local regime, the full line is result of the two fluid model.

to the layers $\lambda_{\perp}$ will contribute to the total frequency shift. The frequency shift due to the specimen is given by

$$
\frac{\Delta f}{f_{0}}=\frac{1}{2} \frac{H_{0}^{2}}{\left.<H_{0}^{2}\right\rangle} \frac{S_{\|} \lambda_{\|}+S_{\perp} \lambda_{\perp}}{V}
$$

where $S$ is the surface area of the specimen, $V$ the volume of the cavity, $H_{0}$ the magnetic field at the surface and $\left\langle H_{0}^{2}\right\rangle$ the magnetic field strength squared averaged over the area of the bottom plate of the cavity.

The measured penetration depth in the two directions is displayed in Fig. 9, where the dotted line is the BCS weak coupling, the full line is the two fluid expression $\lambda_{T}(T)=$ $\lambda_{0}\left[1-\left(T / T_{c}\right)^{4}\right]^{-1 / 2}$ (close to $\lambda(T)$ as would be observed for strong coupling), the dashed line represents the local regime. It is clear, that in both directions $\lambda(T)$ is well described by assuming a singlet ground state. While triplet pairing results in a linear temperature dependence, $s$-wave pairing leads to a flat behavior with $\lim _{T \rightarrow 0} d \lambda(T) / d T=0$.

Both parameters can be understood using conventional electrodynamics to the normal and superconducting state. The dc conductivity measured above the transition $\sigma_{d c}(T=12 \mathrm{~K})=3.8 \times 10^{3}(\Omega \mathrm{cm})^{-1}$ can be combined with an intermediate value for the plasma frequency $\nu_{p}=6000 \mathrm{~cm}^{-1}$ to obtain the relaxation rate $1 / \tau=\frac{\omega_{p}^{2}}{4 \pi \sigma_{d c}}$. This leads to $1 / \tau=1000 \mathrm{~cm}^{-1}$, larger than the gap frequency $\nu_{g a p}=2 \Delta=3.5 k_{B} T_{c}=25 \mathrm{~cm}^{-1}$, expected for the weak coupling BCS limit. With these parameters, the $T=0 \mathrm{~K}$ pene- 
tration depth $\lambda(0)$, using the mean free path correction, is given by

$$
\lambda(0)=\lambda_{L}(0)\left[1+\frac{\xi_{0}}{l}\right]^{1 / 2}=\frac{c}{\omega_{p}}\left[1+\frac{1}{\pi^{2} c \tau \nu_{g a p}}\right]^{1 / 2},
$$

where the coherence length $\xi_{0}=\frac{\hbar l}{\pi \tau \Delta}$ and Eq. 3 is applied. With the previous values of $1 / \tau, \Delta$ and $\nu_{p}$ one obtains $\lambda(T=0)=7800 \AA$, in excellent agreement with the measured values of $1 \mu \mathrm{m}$ (Harshman et al. 1990). The same values give $\lambda_{L}(0)=3300 \AA$ for the London penetration depth.

Perpendicular to the plane, the coherence length $\xi_{\perp}<d$, where $d$ is the interplane separation. Under such circumstances, the situation is close to that of Josephson coupled planes, with the resulting penetration depth

$$
\lambda_{\perp}=\left[\frac{\hbar c^{2} \rho_{\perp}}{4 \pi^{2} \Delta}\right]^{1 / 2}
$$

where $\rho_{\perp}$ is the resistivity perpendicular to the planes. Our surface resistance measurements give $\rho_{\perp}=0.25 \Omega \mathrm{cm}$ as an approximate value just above the transition; this, together with $\Delta=1.76 k_{B} T_{c}$ leads to $\lambda_{\perp}=32 \mu \mathrm{m}$ in excellent agreement with the experimentally obtained values.

Next we turn to the complex conductivity $\sigma=\sigma_{1}+i \sigma_{2}$ in the superconducting state which can be evaluated from Equation 9 using both $R_{S}(T)$ and $X_{S}(T)$ as input parameters:

$$
\sigma_{1}=\frac{2 \mu_{0} \omega R_{S} X_{S}}{\left(R_{S}^{2}+R_{S}^{2}\right)^{2}}
$$

and

$$
\sigma_{2}=\frac{\mu_{0} \omega\left(X_{S}^{2}-R_{S}^{2}\right)}{\left(R_{S}^{2}+X_{S}^{2}\right)^{2}}
$$

For a BCS superconductor in the dirty limit, the Equations 4 and 5 , as work out by Mattis and Bardeen (1958), determine the electrodynamic response. As pointed out before, his limit may not be appropriate for $\kappa$-(BEDT-TTF $)_{2}\left[\mathrm{Cu}(\mathrm{NCS})_{2}\right]$, since the mean free path $l=100 \AA$, the coherence length $\xi_{\|}=70 \AA$ (Saito 1989) and the penetration depth $\lambda_{\|}$ $=1 \mu \mathrm{m}$. We have calculated $R_{S}$ and $X_{S}$ for various values of $l / \pi \xi_{0}$, assuming a twodimensional BCS ground state, with a single particle gap given by the weak-coupling limit $2 \Delta(T=0 \mathrm{~K})=3.5 k_{B} T_{c}$. The best fit of our $60-\mathrm{GHz}$ data leads to a value of $l / \pi \xi_{\|}$ $=1$, and consequently to $\omega \tau_{\|}=0.15$ at $f=\omega / 2 \pi=60 \mathrm{GHz}$. For the relaxation rate we obtain $1 / \tau_{\|}=15 \mathrm{~cm}^{-1}$. In order to normalize the conductivity to the normal state value, we assume a temperature dependent scattering rate yielded from a fit of the normal state resistivity above $T_{c}$, and extrapolate this behavior below the transition. In Fig. 10a we display both components of the complex conductivity $\sigma_{1}$ and $\sigma_{2}$ in the highly conducting (bc)-plane, normalized to the normal state value at $T=9 \mathrm{~K}$. The same parameters are plotted in Figure $10 \mathrm{~b}$ for the direction perpendicular to the plane. In the later case the best fit was obtained with $l / \pi \xi_{\perp}=2$, where $\xi_{\perp}=5 \AA$ is perpendicular coherence length (Saito 1989).

The first obvious feature of Fig. 10 is that the normalized behavior is highly similar in both orientations, parallel to the $(b c)$-plane as well as perpendicular to it, despite the 

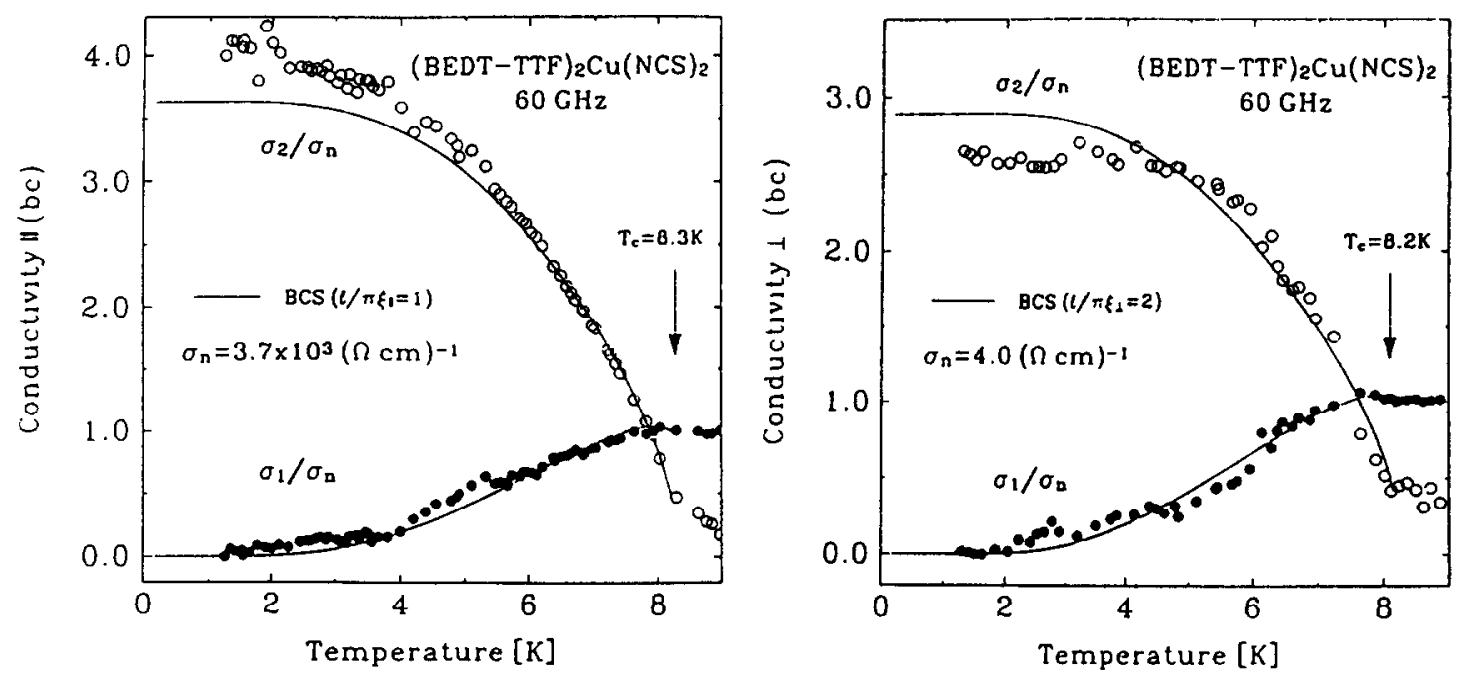

Figure 10: Temperature dependence of the normalized components $\sigma_{1}$ and $\sigma_{2}$ of the optical conductivity at $60 \mathrm{GHz}$ both (a) parallel and (b) perpendicular to the highly conducting layers. The solid lines represent the results of the BCS theory with $l / \pi \xi=1$ and 2 , respectively.

fact that the normal state values are several orders of magnitude different between the two configurations. Both of our results are in full agreement with a BCS ground state. The small coherence peak in the $\sigma_{1}$-curve finds a natural explanation in the BCS model: the broad maximum reflects the case-II coherence factor (Tinkham 1975). At $60 \mathrm{GHz}$ the photon energy is comparable to the single particle gap $\Delta$, and the peak is almost smeared out. Recent experiments at $35 \mathrm{GHz}$ exhibit a peak value of $\sigma_{1} / \sigma_{n} \approx 1.35$, again in the excellent agreement with the BCS theory (Dressel et al. 1993). We note that higher-momentum pairing leads to the rapid disappearance of the coherence peak and is expected to give $\sigma_{1}$ values significantly below the solid line in Fig. 10. The size of the coherence peak depends on the coherence factor plus the divergency of the excitation density of states at the the gap edge. It is expected that this singularity is removed for $d$-wave pairing leading to a sharp decrease of the optical conductivity analogous to the Yosida function. For $s$-wave pairing, the photon energy smooths out this singularity and is responsible for the reduced height of the peak feature for this relatively-small $T_{c}$ compound (the size of the peak goes as $\log \frac{\hbar \omega}{\Delta}$ ). As a consequence, since we oberserve the same temperature dependence for $\sigma_{1}(T)$, we deduce that the divergency in the optical response of the system is identical at various orientations. We conclude that the ratio $\Delta / \hbar \omega$ is a fundamental constant of the superconducting phase and thus the gap is isotropic for all three crystallographic axes. For $d$-wave pairing the gap should have vanished along the $a$-direction. We conclude, that the temperature dependence of $\sigma_{1}(T)$ in both orientations, as shown in Fig. 10, is another independent evidence, besides the temperature dependence of the penetration depth $\lambda(T)$, that the pairing is indeed $s$-wave in $\kappa$-(BEDT-TTF $)_{2}\left[\mathrm{Cu}(\mathrm{NCS})_{2}\right]$ and related materials. 


\section{Conclusions}

Our experiments suggest that the normal state properties of both $\kappa$-(BEDT-TTF $)_{2}[\mathrm{Cu}$ $\left.(\mathrm{NCS})_{2}\right]$ and $\mathrm{X}_{3} \mathrm{C}_{60}$ are that of a Drude metal with a moderately enhanced effective mass. More detailed experiments are required to search for deviations from conventional Fermi-liquid behavior. We also find, in both cases, a MIR absorption, arriving most probably from band structure effects. The superconducting state is that of the conventional $s$-wave as evidenced by the temperature dependence of the penetration depth, and also by the conductivity coherence effects, observed near $T_{c}$, with strongly anisotropic parameters in the layered compound (BEDT-TTF $)_{2}\left[\mathrm{Cu}(\mathrm{NCS})_{2}\right]$. The measured gaps in the superconducting $\mathrm{C}_{60}$ compounds suggest weak coupling with $2 \Delta / k_{B} T_{c}$ close to the BCS value.

\section{Acknowledgements}

We wish to thank F. Wudl and H. Yamochi, J.M. Williams, K.D. Carlson and H.H. Wang for providing the BEDT-TTF samples. The $\mathrm{X}_{3} \mathrm{C}_{60}$ compounds were synthesized by $\mathrm{R}$. Kaner, S.-M. Huang and J. Wiley. This work was supported by the INCOR program of the University of California and by DARPA. One of us (M.D.) acknowledges support from the Alexander von Humboldt Foundation. L.D. wishes to thank for the financial support of the Swiss National Foundation for Scientific Research.

\section{References}

L. DeGiorgi, G. Grüner, P. Wachter, S.-M. Huang, J. Wiley, R.L. Whetten, R.B. Kaner, K. Holzcer and F. Diederich, Phys. Rev. B 46 (1992), 11250

L. DeGiorgi, P. Wachter, G. Grüner, S.-M. Huang, J. Wiley and R.B. Kaner, Phys. Rev. Lett. 69 (1992), 2987

M. Dressel, S. Bruder, G. Grüner, K.D. Calson, H.H. Wang and J.M. Williams, (to be published 1993)

R.C. Haddon, A.F. Hebard, M.J. Rosseinsky, D.W. Murphy, S.J. Duclos, K.B. Lyons, B. Miller, J.M. Rosamilia, R.M. Fleming, A.R. Kortan, S.H. Glarum, A.V. Makhaija, A.J. Muller, R.H. Eick, S.M. Zahurak, R. Tycko, G. Dabbagh and F.A. Thiel, Nature (London) 350 (1991), 320

D.R. Harshman, R.N. Kleiman, R.C. Haddon, S.V. Chichester-IIicks, M.L. Kaplan, L.W. Rupp, T. Pfiz, D.L. Williams and D.B. Mitzi, Phys. Rev. Lett. 64 (1990), 1293

K. Holczer, O. Klein, G. Grüner, H. Yamochi and F. Wudl, in: Organic Superconductivity, ed. V.Z. Kresin and W.A. Little, Plenum Press, New York 1990

K. Holczer, O. Klein, G. Grüner, J.D. Thompson, F. Diederich and R.L. Whetten, Phys. Rev. Lett. 67 (1991), 271

M.-Z. Huang, Y.-N. Xu and W. Y. Ching, Phys. Rev. B 46 (1992), 6572

D. Jung, M. Evain, J.J. Novoa, M.-H. Whangbo, M.A. Beno, A.M. Kini, A.J. Schultz, J.M. Williams and P.J. Nigrey, Inorg. Chem. 28 (1989), 4516

K. Kanoda, K. Akiba, K. Suzuki and T. Takahashi, Phys. Rev, Lett. 65 (1990), 1271

S. Katsumoto, S. Kobayashi, H. Urayama, H. Yamochi and G. Saito, J. Phys. Soc. Jpn. 57 
(1988), 3672

O. Klein, K. Holczer and G. Grüner, J.J. Chang and F. Wudl, Phys. Rev. Lett. 66 (1991), 655

O. Klein, G. Grüner, S.-M. Huang, J.B. Wiley and R.B. Kaner, Phys. Rev, B 46 (1992), 11247

K. Kornelsen, J.E. Eldridge, C.C. Homes, H.H. Wang and J.M. Williams, Solid State Commun. $72(1989), 475$

K. Kornelsen, J.E. Eldridge, H.H. Wang and J.M. Williams, Solid State Commun. 76 (1990), 1009

K. Kornelsen, J.E. Eldridge, H.H. Wang and J.M. Williams, Phys. Rev. B 44 (1991), 5235

L.P. Le, G.M. Luke, B.J. Sternlieb, W.D. Wu, Y.J. Uemura, J.H. Brewer, T.M. Riseman, C.E. Stronach, G. Saito, H. Yamochi, H.H. Wang, A.M. Kini, K.D. Carlson and J.M. Williams, Phys. Rev. Lett. 68 (1992), 1923

D.C. Mattis and J. Bardeen, Phys. Rev, 111 (1958), 412

K. Oshima,, H. Urayama, H. Yamochi and G. Saito, J. Phys. Soc. Jpn. 57 (1988), 730

T.T.M. Palstra, R.C. Haddon, A.F. Hebbard and J. Zaanen, Phys. Rev. Lett. 68 (1991), 1054

G. Saito, Physica C 162-164 (1989), 577

G. Saito, Mol. Cryst. Liq. Cryst. 181 (1990), 65

E. Sohmen, J. Fink and W. Kraetschmer, Europhys. Lett. 17 (1992), 51

E. Sohmen and J. Fink, (to be published 1993)

G. Sparn, J.D. Thompson, R.L. Whetten, S.-M. Huang, R.B. Kaner, F. Diederich, G. Grüner and K. Holczer, Phys. Rev. Lett. 68 (1992), 1228

S. Tajima, S. Uchida, M. Madaki, H. Takagi, K. Kitazawa and S. Tanaka, Phys. Rev. B 32 (1985), 6302

T. Takahashi, T. Tokiwa, K. Kanoda, H. Urayama, H. Yamochi and G. Saito, Physica C 153-155 (1988), 487

T. Takahashi, S. Suzuki, T. Morikawa, K. Katayama-Yoshida, S.H. Asegawa, H. Inokuchi, K. Seki, K. Kikuchi, S. Suzuki, K. Ikemoto and Y. Achiba, Phys. Rev. Lett. 68 (1992), 1232

D.B. Tanner and T. Timusk, in: Physical Properties of High Temperature Superconductors III, ed. D.M. Ginsberg, World Scientific, Singapore 1992

M. Tinkham, Introduction to Superconductivity, McGraw-Hill, New York 1975

N. Toyota, T. Sasaki, K. Murata, Y. Honda, M. Tokumoto, H. Bando, N. Kinoshita, H. Anzai,

T. Ishiguro and Y. Muto, J. Phys. Soc. Jpn. 57 (1988), 2616

R. Tycko, G. Dabbagh, M.J. Rosseinsky, D.W. Murphy, A.P. Ramirez, R.M. Flemming, Phys.

Rev. Lett. 68 (1992), 1912

Y.T. Uemura, A. Keren, L.P. Le, G.M. Luke, B.J. Sternlieb, W.D. Wu, J.B. Brewer, R.L. Whetten, S.M. Huang, S. Lin, R.B. Kaner, F. Diederich, S. Donovan, G. Grüner and K. Holczer, Nature (London) 352 (1991), 605

Y.T. Uemura, L.P. Le and G.M. Luke, in Proceedings of the International Conference on Science and Technology of Synthetic Metals, Göteborg, Sweden 1992, [Synth. Met. (to be published 1993)]

J.M. Williams, J.R. Ferraro, R.J. Thorn, K.D. Carlson, U. Geiser, H.H. Wang, A.M. Kini and M.-H. Whangbo, Organic Superconductors, Prentice Hall, Englewood Cloffs NJ 1992

W.H. Wong, M. Hanson, W.G. Clark, K. Holczer, G. Güner, J.D. Thompson, R.L. Whetten, S.-M. Huang, R.B. Kaner and F. Diederich, Europhys. Lett. 18 (1992), 79

X.D. Xiang, J.G. Hou, G. Briceno, W.A. Vareka, R. Mostovoy, A. Zettl, V. Crespi and M.L. Cohen, Science 256 (1992), 1190

Y.-N. Xu, M.-Z. Huang and W.Y. Ching, Phys. Rev. B 44 (1991), 13171 